\title{
Partial Characterization of Mycobacterium fortuitum and Mycobacterium smegmatis Auxotrophs by Syntrophism Using Bacillus subtilis
}

\author{
By V. R. SUBRAMANYAM* AND K. K. MOHANTY \\ Regional Medical Research Centre, Indian Council of Medical Research, PO Sainik School, \\ Bhubaneswar 751 005, India
}

(Received 6 April 1989; revised 12 June 1989; accepted 22 June 1989)

\begin{abstract}
Syntrophism (cross-feeding) could be demonstrated between mutants of Mycobacterium fortuitum and Mycobacterium smegmatis, and previously characterized mutants of Bacillus subtilis, auxotrophic for arginine, histidine, lysine or phenylalanine. Based on this cross-feeding data, the possible site of blockage in the biosynthetic pathways of the mutants could be inferred.
\end{abstract}

\section{INTRODUCTION}

Most mycobacteria can be cultured in simple media and should therefore be capable of de novo biosynthesis of precursors of macromolecules. For a better understanding of the peculiarities of the genus Mycobacterium, it is necessary to study the relevant biosynthetic pathways. Not much information is available on the basic biology of Mycobacterium, owing to the lack of a wellworked-out system for genetic manipulation in this genus. Only recently have there been reports of concerted efforts for cloning genes from and into mycobacteria (Young et al., 1985; Hopwood et al., 1988; Snapper et al., 1988). This approach would help, in no small way, our understanding of mycobacteria. However, while molecular cloning is a valuable tool, the importance of work on classical genetics cannot be over-emphasized.

Syntrophism or cross-feeding is a classical method in biochemical genetics for obtaining information on the affected sites in a biosynthetic pathway. Auxotrophs with a mutation at a particular step in a biosynthetic pathway cross-feed mutants blocked at an earlier stage in that pathway. Such an approach has so far not been reported for studying biosynthetic pathways in Mycobacterium. We isolated several auxotrophic mutants of Mycobacterium spp. (Subramanyam et al., 1989) and in this work, we have characterized some of the mutants by syntrophism. Nonavailability of well-characterized mutants of Mycobacterium prompted us to use mutants of Bacillus subtilis, which have well-characterized mutations and are easily available as reference strains.

\section{METHODS}

Organisms and media. The strains used in this study are listed in Table 1. Nutrient agar (NA), Middlebrook 7H9 broth base (MB) and agar were from HiMedia Laboratories, Bombay; amino acids and cytosine were from LobaChemie Indoaustranal Co., Bombay. MB without ADC (albumin/dextrose/catalase) enrichment but with glucose $(0.5 \% \mathrm{w} / \mathrm{v})$, glycerol $(0.2 \% \mathrm{v} / \mathrm{v})$ and agar $(1.5 \% \mathrm{w} / \mathrm{v})$ was used as basal medium.

Syntrophism. To look for cross-feeding with reference to a particular amino acid, strains with similar amino acid requirements were tested against each other by streaking on basal medium supplemented with a suboptimal concentration $\left(1 \mu \mathrm{g} \mathrm{ml}^{-1}\right)$ of the amino acid. When using strains with multiple requirements, all the required nutrients, except the one under test, were provided $\left(20 \mu \mathrm{g} \mathrm{ml}^{-1}\right)$ in the basal medium. For example, when strain $1 \mathrm{~A} 8$ was used for testing syntrophism of lysine auxotrophs, the basal medium was supplemented with $20 \mu \mathrm{g}$ aromatic amino acids $\mathrm{ml}^{-1}$, and only $1 \mu \mathrm{g}$ lysine $\mathrm{ml}^{-1}$. Because of the difference in growth rates, $B$. subtilis strains were inoculated $24 \mathrm{~h}$ after the mycobacteria. Three strains per plate were tested in different combinations. Plates were read after $2-4 \mathrm{~d}$ incubation at $37^{\circ} \mathrm{C}$. Because of the low concentration $\left(1 \mu \mathrm{g} \mathrm{ml}^{-1}\right)$ of the required amino acid in the media, auxotrophic mutants do not show appreciable growth except when cross-fed by a neighbouring strain. Thus, a better growth of mutant ' $X$ ' in the proximity of mutant ' $Y$ ' was recorded as positive cross-feeding in this instance ' $Y$ ' cross-feeds ' $X$ ' (see Fig. 1). 
Table 1. Strains used

\begin{tabular}{|c|c|c|}
\hline Strain & Markers* & Source or derivation $\dagger$ \\
\hline \multicolumn{3}{|l|}{ B. subtilis } \\
\hline $1 \mathrm{~A} 8$ & $\operatorname{trpC2}$ aroD120 lys-1 & BGSC \\
\hline $1 \mathrm{~A} 10$ & $\operatorname{trpC2}$ his Al thr-5 & BGSC \\
\hline $1 \mathrm{~A} 96$ & $\operatorname{trpC} 2$ pheAI & BGSC \\
\hline $1 \mathrm{~A} 510$ & leuA8 arg 15 thrA recE4 $r^{-} m^{-}$stp & BGSC \\
\hline \multicolumn{3}{|l|}{ M. fortuitum } \\
\hline NIHJ 1615 & Prototroph & NIHJ \\
\hline SM2 & arg-1 & NTG treatment of NIHJ 1615 \\
\hline SM29 & arg-1 leu-2 his-1 & NTG treatment of SM2 \\
\hline \multicolumn{3}{|l|}{ M. smegmatis } \\
\hline NIHJ1628 & Prototroph & NIHJ \\
\hline SM10 & lys-1 & NTG treatment of NIHJ 1628 \\
\hline SM13 & leu-1 & NTG treatment of NIHJ1628 \\
\hline SM20 & lys-2 & NTG treatment of NIHJ 1628 \\
\hline SM22 & $\arg -2$ & NTG treatment of NIHJ 1628 \\
\hline SM33 & leu-1 cyt-1 arg-3 & NTG treatment of SM13 \\
\hline SM42 & lys-I phe-1 & NTG treatment of SM 10 \\
\hline
\end{tabular}

* Relevant characteristics of $B$. subtilis used as reference strains: lys-1, blocked in diaminopimelate decarboxylase; his $A 1$, histidine requirement; pheAl, blocked in prephenate dehydratase; leuA8, blocked in $\alpha$-isopropylmalate synthase; $\arg 15$, arginine requirement.

† All B. subtilis strains were obtained from the Bacillus Genetic Stock Center (BGSC), The Ohio State University, USA; NIHJ1615 and NIHJ1628 were from the National Institute of Health (NIHJ), Japan; all strains with the prefix SM have been derived in our laboratory by mutagenesis of NIHJ strains with $N$-methyl- $N^{\prime}-$ nitro- $N$-nitrosoguanidine (NTG), as described elsewhere (Subramanyam et al., 1989). (Briefly, bacteria were treated with $500 \mu \mathrm{g} \mathrm{NTG} \mathrm{ml} \mathrm{m}^{-1}$ at $37^{\circ} \mathrm{C}$ for $30 \mathrm{~min}$, washed and plated on nutrient agar. Auxotrophs were identified by replica plating onto minimal medium. After three subcultures, only a fraction of the mutants retained their auxotrophic trait.)

\section{RESULTS AND DISCUSSION}

For Mycobacterium, there is no information at all on the biosynthesis of arginine, glycine, histidine, methionine, proline, serine and threonine (Ratledge, 1982). In the biosynthesis of lysine, only the final step has been studied (Willett, 1969). Regarding the biosynthesis of aromatic amino acids there have been only two reports, one showing the presence of tryptophan synthetase in $M$. smegmatis (Ratledge \& Winder, 1961) and the other demonstrating all six enzymes of the common pathway in M. phlei (Yapo et al., 1974).

Use of reference strains of bacteria such as $B$. subtilis offers great potential for understanding more about mycobacterial mutants. Both interspecies and intergeneric cross-feeding was observed amongst mutants of $M$. fortuitum, $M$. smegmatis, and $B$. subtilis auxotrophic for arginine, histidine, lysine and phenylalanine (Table 2). There was no cross-feeding amongst leucine auxotrophs.

Based on the cross-feeding data, the mutants could be arranged sequentially according to the possible site of mutation in the biosynthetic pathway (Table 3). In the pathway for arginine biosynthesis, by analogy with Escherichia coli (Vogel, 1970), SM2 is blocked in the final steps of formation of arginine, either at the argininosuccinate synthetase $(\arg G)$ or at the argininosuccinase $(\arg H)$ level; SM33 is suspected to be a mutant either at carbamoylphosphate formation or at the ornithine carbamoyltransferase $(\arg F)$ locus, and SM22 at a locus preceding $\arg F$. These inferences are corroborated by the growth patterns of the mutants on basal medium supplemented with ornithine or citrulline, which are intermediates in arginine biosynthesis. SM22 grows equally well on ornithine, citrulline or arginine; SM33 grows on citrulline or arginine, but not on ornithine; and SM2 and 1 A510 grow only on arginine but not on ornithine or citrulline.

In histidine biosynthesis, SM29 is expected to be blocked downstream of his A since SM29 can cross-feed $1 \mathrm{~A} 10$ which has a mutation at his $A$. Similarly, cross-feeding data indicate that in 


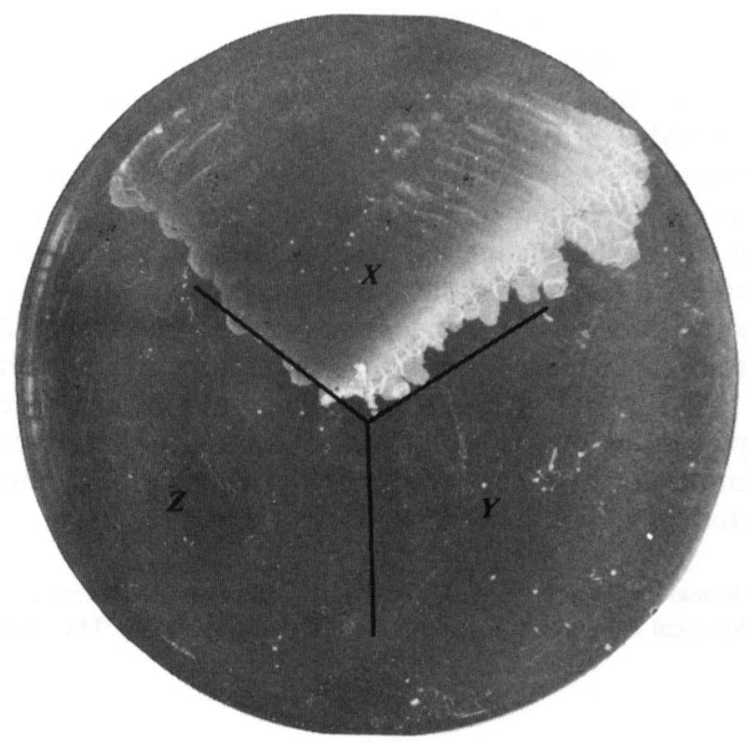

Fig. 1. Syntrophism (cross-feeding). Mutants ' $X$ ' (SM22), ' $Y$ ' (1A510) and ' $Z$ ' (SM2) were streaked on a minimal medium supplemented with only $1 \mu \mathrm{g} \mathrm{ml}^{-1}$ of the required amino acid (arginine). However, both ' $Y$ ' and ' $Z$ ' are able to cross-feed ' $X$ ' ' $X$ ' shows good growth only in the proximity of the other two mutants.

Table 2. Cross-feeding between auxotrophs of $M$. fortuitum, M. smegmatis and B. subtilis

Cross-feeding with reference to each amino acid was examined as described in Methods by testing all combinations of the strains auxotrophic for that amino acid. Strains listed under column I cross-feed corresponding strains of column II on a medium supplemented with $1 \mu \mathrm{g} \mathrm{ml}^{-1}$ of the amino acid shown in column III. SM2 and SM29 are mutants of $M$. fortuitum; SM10, SM20, SM22, SM33 and SM42 are mutants of $M$. smegmatis; $1 \mathrm{~A} 8,1 \mathrm{~A} 10,1 \mathrm{~A} 96$ and $1 \mathrm{~A} 510$ are reference $B$. subtilis mutants. Combinations not shown in the Table were negative in cross-feeding experiments. All combinations were tested on four different occasions, with the same result.

$$
\text { I II III }
$$

$\begin{array}{lll}\text { SM2 } & \text { SM22 and SM33 } & \begin{array}{l}\text { Arginine } \\ \text { Arginine }\end{array} \\ \text { SM33 } & \text { SM22 } & \text { Arginine } \\ \text { 1A510 } & \text { SM22 and SM33 } & \text { Histidine } \\ \text { SM29 } & \text { 1A10 } & \text { Lysine } \\ \text { SM20 } & \text { SM10 } & \text { Lysine } \\ \text { 1A8 } & \text { SM10 and SM20 } & \text { Phenylalanine } \\ \text { SM42 } & 1 \text { A96 } & \end{array}$

Table 3. Mutants arranged according to the possible site of mutation in the biosynthetic pathway based on cross-feeding data

\begin{tabular}{ll} 
Biosynthesis of: & \multicolumn{1}{c}{ Mutants* } \\
Arginine & SM22 $\rightarrow$ SM33 $\rightarrow$ SM2 and 1A510 \\
Histidine & 1A10 $\rightarrow$ SM29 \\
Lysine & SM10 $\rightarrow$ SM20 $\rightarrow$ AA8 \\
Phenylalanine & 1A96 $\rightarrow$ SM42
\end{tabular}

* Relative positions from left-to-right indicate early-to-later steps in biosynthesis. SM2 and SM29 are $M$. fortuitum mutants; SM10, SM20, SM22, SM33 and SM42 are M. smegmatis mutants; 1A8, 1A10, 1A96 and 1 A510 are reference $B$. subtilis mutants.

† SM2 and 1A510 probably each have a mutation at the same step. 
lysine biosynthesis, SM10 and SM20 are blocked at two different points (SM10 at an earlier step than SM20), preceding the final step of L-lysine formation from meso-diaminopimelate, because $1 \mathrm{~A} 8$, which is blocked at this final step, cross-feeds both SM20 and SM10, and SM20 in turn cross-feeds SM10. Since SM42 cross-feeds 1 A96 which lacks prephenate dehydratase (the enzyme catalysing the penultimate step in the biosynthesis of phenylalanine), it can be inferred that SM42 is blocked at the very final step in phenylalanine biosynthesis.

Lack of cross-feeding among the leucine-requiring mutants suggests a common biosynthetic block, possibly at the level of $\alpha$-isopropylmalate synthase, because 1 A510 is a known mutant at this site (leuA8). It is important to note that several factors affect the results of cross-feeding experiments. The results might be misleading if, for instance, the strains being tested have other than point mutations in structural genes. Hence the results of cross-feeding experiments, particularly where only negative results are obtained, need to be interpreted with caution.

These findings indicate that using more reference strains of Bacillus and perhaps E. coli, it should be possible to study the biochemical genetics of Mycobacterium.

We acknowledge the technical assistance of Mr B. B. Pal and the encouragement given by Professor L. N. Mohapatra. The Regional Medical Research Centre, Bhubaneswar is funded by The Indian Council of Medical Research.

\section{REFERENCES}

Hopwood, D. A., Kieser, T., Colston, M. J. \& LAMb, F. I. (1988). Molecular biology of mycobacteria. British Medical Bulletin 44, 528-546

RATLEDGE, C. (1982). Nutrition, growth and metabolism. In The Biology of the Mycobacteria, vol. 1, pp. 185-271. Edited by C. Ratledge \& J. L. Stanford. London: Academic Press.

Ratledge, C. \& Winder, F. G. (1961). Tryptophan synthetase in Mycobacterium smegmatis. Biochemical Journal 80, 20-21.

SNAPPER, S. B., Lugosi, L., JekKel, A., Melton, R. E., KieSer, T., BloOM, B. R. \& JACOBS, W. R., JR (1988). Lysogeny and transformation in mycobacteria: stable expression of foreign genes. Proceedings of the National Academy of Sciences of the United States of America 85, 6987-6991.

Subramanyam, V. R., Pal, B. B. \& Mohanty, K. K. (1989). Inducibility and stability of auxotrophic mutations in Mycobacterium fortuitum, M. smegmatis and $M$. vaccae. Letters in Applied Microbiology 8 , 161-164.

VoGEL, H. J. (1970). Arginine biosynthetic system in Escherichia coli. Methods in Enzymology 17A, 249-251.

WILLETT, H. P. (1969). The production of lysine from diaminopimelic acid by cell free extracts of $\mathrm{Myco}$ bacterium paratuberculosis. American Review of Respiratory Diseases 81, 653-659.

YaPo, A., Catala, F. \& Azerad, R. (1974). Separability of enzymes of the common aromatic biosynthetic pathway in Mycobacterium phlei. Biochemie 56, $1145-1146$.

Young, R. A., Bloom, B. R., Grossinsky, C. M., IVANYI, J., Thomas, D. \& Davis, R. W. (1985). Dissection of Mycobacterium tuberculosis antigens using recombinant DNA. Proceedings of the National Academy of Sciences of the United States of America 82, 2583-2587. 